\title{
Magnetoresistance due to domain walls in an epitaxial microfabricated Fe wire
}

\author{
U. Rüdiger, ${ }^{\text {a) }}$ J. Yu, and A. D. Kent ${ }^{\text {b) }}$ \\ Department of Physics, New York University, New York, New York 10003
}

S. S. P. Parkin

IBM Almaden Research Center, San Jose, California 95120

(Received 23 March 1998; accepted for publication 6 July 1998)

\begin{abstract}
The domain wall (DW) contribution to magnetoresistance has been investigated using an epitaxial microfabricated bcc (110) Fe wires of $2 \mu \mathrm{m}$ linewidth. A strong in-plane uniaxial component to the magnetic anisotropy perpendicular to the wire axis causes a regular stripe domain pattern with closure domains. The stripe domain width in zero-applied magnetic field is strongly affected by the magnetic history and can be continuously varied from 0.45 to $1.8 \mu \mathrm{m}$. This enables a measurement of the resistivity as a function of DW density in a single wire. Clear evidence is presented that the resistivity is reduced in the presence of DWs at low temperatures.
\end{abstract}

The miniaturization of magnetoelectronic devices requires controlling domain configurations in small ferromagnetic elements and understanding the effect of domain walls (DWs) and sample boundaries on electronic transport in these elements. Of particular recent interest is the possibility that DWs can have a significant effect on spin-dependent electron transport. ${ }^{1-4}$ However, the study of DW resistivity requires isolating this effect from ordinary anisotropic magnetoresistance (AMR) in which the resistivity depends on the relative orientation of the current $\mathbf{j}$ and magnetization $\mathbf{M}$ and the anisotropy of the Lorentz magnetoresistance (MR), which generally depends on the angle of the current $\mathbf{j}$ and internal magnetic-field $\mathbf{B}$. Since applying a magnetic field to a multidomain sample changes the domain configurations and orientations, this is a source of MR and can be a large effect. $^{5,6}$ Here, an approach is employed to isolate a DWrelated contribution to the resistivity of a single microfabricated Fe wire in which the magnetic stripe domain configuration and DW density can be continuously varied.

Important to these experiments is controlling magnetic interactions in a microfabricated wire. First, bcc (110) Fe films were grown on $a$-axis sapphire substrates using ultrahigh-vacuum e-beam evaporation techniques. ${ }^{7-9}$ A $10 \mathrm{~nm}$ thick (110) Mo seed layer was deposited at $900 \mathrm{~K}$ at a rate of $0.2 \mathrm{~nm} / \mathrm{s}$ followed by a $100 \mathrm{~nm}$ thick (110) Fe layer grown at $510 \mathrm{~K}$ at a rate of $0.5 \mathrm{~nm} / \mathrm{s}$. Longitudinal Kerr-hysteresis measurements indicate a strong uniaxial magnetocrystalline component to the anisotropy with an in-plane easy axis along the [001] direction. X-ray $\Theta / 2 \Theta$ scans indicate a fully (110) orientation of the Fe films and $\mathrm{X}$-ray pole figures show that the in-plane [1 $\overline{1} 1]$ axis of the films is parallel to the [0001] direction of the sapphire substrate. The residual resistivity ratio of 30 and the residual resistivity of $\rho_{0}=0.2 \mu \Omega \mathrm{cm}$ confirm the high crystalline quality of the films. Using optical projection lithography techniques and ion milling, $\mathrm{Fe}$

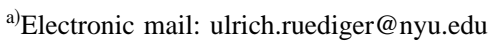

${ }^{b)}$ Electronic mail: andy.kent@nyu.edu
}

wires of $2.0 \mu \mathrm{m}$ linewidth were fabricated with the magnetic easy axis perpendicular to the wire axis.

The competing magnetostatic, magnetocrystalline, and exchange interactions result in a regularly spaced stripe domain configuration with closure domains. ${ }^{10}$ By minimizing the free energy of this configuration it is easily seen that the domain width should depend on the square root of the wire width. ${ }^{10}$ However, magnetic force microscope (MFM) images at room temperature reveal the presence of metastable domain configurations. The MFM images of a $2 \mu \mathrm{m}$ wire shown in Figs. 1(a)-1(d) demonstrate a strong influence of the prior saturation field direction on the stripe domain width. Here, by using a magnetic tip with a remanent magnetization perpendicular to the wire surface, the MFM images highlight the out-of-plane component of the wire magnetization, i.e., Bloch walls and magnetic poles at the wire edges. Before performing a MFM image in zero-applied field the wire was magnetized to saturation (a) longitudinal $(\Theta$ $=0^{\circ}$ ), (b) $\Theta=60^{\circ}$, (c) $\Theta=72^{\circ}$, and (d) transverse $(\Theta$ $=90^{\circ}$ ) to the wire axis. Figure 2 shows a continuous increase of the stripe domain width from 0.45 to $1.8 \mu \mathrm{m}$ as function of the angle $\Theta$ between the applied field and the wire axis.

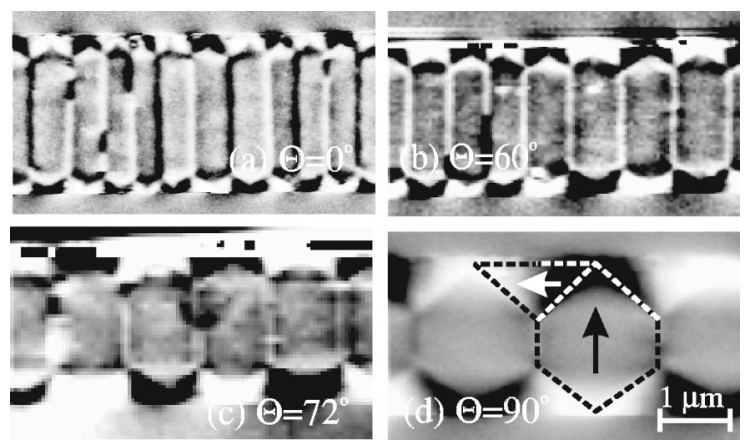

FIG. 1. The MFM images of the stripe domain pattern of a $2 \mu \mathrm{m}$ Fe wire in zero-applied field show a strong dependence of the previous saturation direction. Before performing the MFM images the wire was magnetized to saturation (a) longitudinal $\left(\Theta=0^{\circ}\right)$, (b) $\Theta=60^{\circ}$, (c) $\Theta=72^{\circ}$, and (d) transverse $\left(\Theta=90^{\circ}\right)$, with respect to the wire axis. 


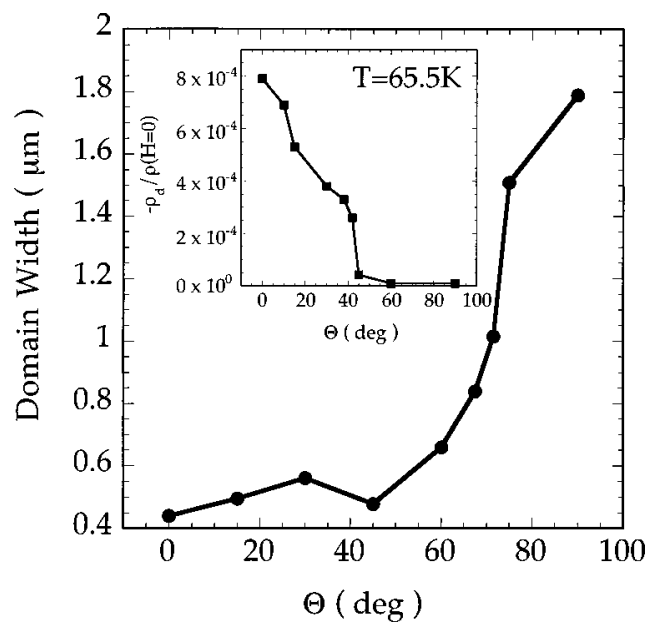

FIG. 2. The domain width in zero-applied field determined from MFM images increases continuously as function of the previous saturation direction labeled by the angle $\Theta$ between the wire axis and the applied field. The inset shows the reduction of resistivity $-\rho_{d} / \rho(H=0)$ due to the presence of DWs as a function of $\Theta$.

In order to examine the large difference in stripe domain width between the longitudinal and transverse geometry, MFM measurements were carried out in fields just below the saturation fields for these configurations. In the longitudinal case shown in Fig. 3(a), besides the triangle-shaped closure domains with the magnetization parallel to the applied field $(H=0.013 \mathrm{~T})$, stripe domains of alternating magnetization direction perpendicular to the field are nucleated. A further reduction of the applied field does not lead to a change of the stripe domain width, only a contraction of closure domains occurs via DW movement. In contrast, the transverse case just below the saturation field $(H=0.03 \mathrm{~T})$ shown in Fig. 3 (b) is dominated by a stripe domain configuration of alternating magnetization direction, where the antiparallel oriented domain is smaller in volume. As in the longitudinal case, a further reduction of the field leads to a DW movement but not further nucleation of DWs. In both instances the nucleation of DWs is associated with the formation of domains oriented perpendicular to the applied magnetic field. In the longitudinal case, these domains are along the magne-

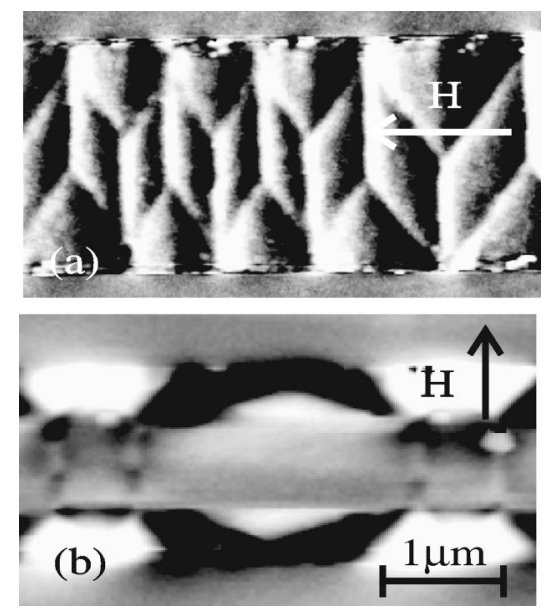

FIG. 3. MFM images of the domain configurations of a $2 \mu \mathrm{m}$ Fe wire in the presence of (a) a longitudinal field of $H=0.013 \mathrm{~T}$ and (b) a transverse field of $H=0.03 \mathrm{~T}$, which are both just below the saturation field of the given orientation. tocrystalline easy axis. However, in the transverse case they are perpendicular to the magnetocrystalline easy axis. Thus, qualitatively, the lower DW density in the transverse case is due to the larger magnetocrystalline energy density associated with the magnetic state after DW nucleation.

MR measurements were performed in a high-field variable temperature cryostat with sample rotation capabilities. The applied field was in-plane and oriented either longitudinal $(\|)$ or transverse $(\perp)$ to the wire axis. The resistivity was measured using a four-probe ac $(\sim 10 \mathrm{~Hz})$ bridge technique with low currents $(10 \mu \mathrm{A})$. In previous experiments we examined the DW contribution to the MR by measuring the low-field MR as a function of temperature, angle of the applied field, and wire linewidth. ${ }^{7,8}$ In this prior work the DW density was varied by varying the wire linewidth. Here, the dependence on DW density is studied more elegantly by varying the DW density in a single $2 \mu \mathrm{m}$ wire. In this manner effects associated with changing the wire cross section or impurity concentration can definitively be isolated from those associated with domain configurations and DWs.

The resistivity of a ferromagnetic metal in general depends on the relative orientation of the magnetization and current (as well as the direction of current in crystalline metals). Since at $H=0$ these wires contain domains oriented both parallel (closure domains) and perpendicular (stripe domains) to the current direction, the observed MR can result from both resistivity anisotropy (Lorentz MR and AMR) as well as the erasure of DWs. MR measurements at room temperature can be entirely explained by resistivity anisotropy. ${ }^{7}$ At $H=0$ the MR in transverse and longitudinal geometry is governed by the resistivity anisotropy and reflects the volume ratio of longitudinal to transverse domains, which was determined by MFM measurements [see Figs. 1(a) and 1(d)]. Besides this resistivity anisotropy, no additional contribution could be detected. ${ }^{7}$ This result on Fe wires is in contrast to $\mathrm{Co}$ and $\mathrm{Ni}$ films, where at room temperature an increased resistivity in the presence of DW was observed. ${ }^{1,2}$

We have found, however, that at lower temperatures the resistivity anisotropy $\left[\rho_{\|}\left(H=0, T_{\text {comp }}\right)=\rho_{\perp}\left(H=0, T_{\text {comp }}\right)\right]$ vanishes. This is due to the increasing importance of the resistivity anisotropy associated with the Lorentz MR which is opposite in sign to the AMR. ${ }^{8}$ At this so-called compensation temperature $T_{\text {comp }}$ the effect of domain configurations on the MR also vanishes and the DW contribution to the MR can be isolated. Figure 4 shows the low- and high-field MR in transverse and longitudinal geometry at the compensation temperature of $T=65.5 \mathrm{~K}$. The absence of resistivity anisotropy at $H=0$ at this temperature is suggested by extrapolation from the high-field resistivity above magnetic saturation for transverse (solid line) and longitudinal (dotted line) applied fields. The field dependence above magnetic saturation is due to the ordinary Lorentz MR. ${ }^{8}$ Nevertheless, the measured resistivities in both field geometries at $H=0$ are lower than the extrapolated value and the differences to the extrapolated value are correlated with the DW densities after longitudinal [Fig. 1(a)] and transverse [Fig. 1(d)] saturation. Here, the higher DW density state gives rise to a lower resistivity. To show that this effect depends systematically on the DW density, different DW densities were established by saturating the wires in an in-plane direction tilted by $\Theta$ 


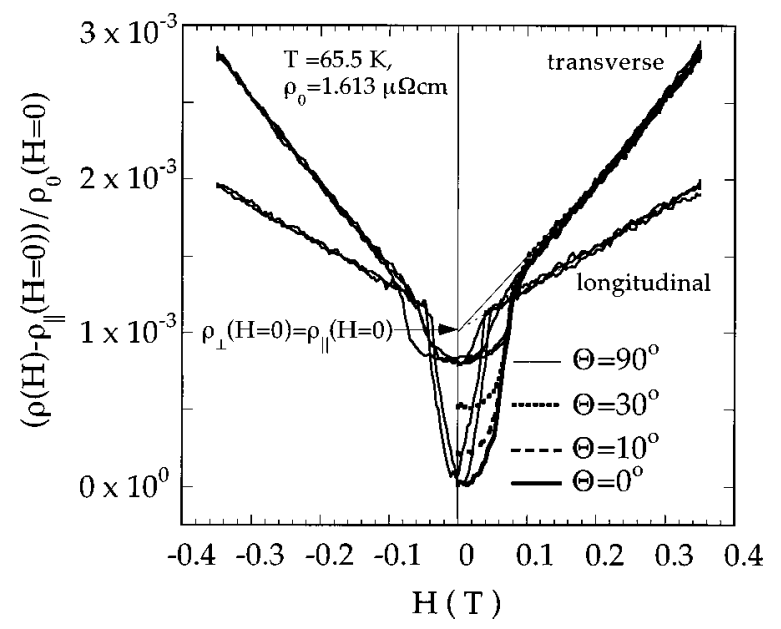

FIG. 4. Low-field MR of a $2 \mu \mathrm{m}$ Fe wire $[\rho(T=65.5 \mathrm{~K})=1.6 \mu \Omega \mathrm{cm}]$ measured at the compensation point temperature of $65.5 \mathrm{~K}$. Extrapolation of the high-field MR data in transverse (solid line) and longitudinal (dotted line) geometry shows the equality of $\rho_{\perp}\left(H=0, T_{\text {comp }}\right)$ and $\rho_{\|}(H$ $\left.=0, T_{\text {comp }}\right)$. Also included are the MR data measured in transverse geometry from $H=0$ to saturation after magnetizing the wire transverse $(\Theta$ $\left.=90^{\circ}\right), \Theta=30^{\circ}, \theta=10^{\circ}$, and longitudinal $\left(\theta=0^{\circ}\right)$, with respect to the wire axis.

$=10^{\circ}$ and $\Theta=30^{\circ}$ with respect to the wire axis. As discussed in the section above for $\Theta=10^{\circ}$, the DW density is higher than for $\Theta=30^{\circ}$. After saturating and before performing the MR measurement, the wire has been oriented in the transverse geometry at $H=0$. The low-field MR data (see Fig. 4) for $\Theta=10^{\circ}$ and $\Theta=30^{\circ}$ show a continuous decrease of the $H=0$ resistivity with respect to the transverse case. These kinds of measurements have been done for a larger set of different angles as plotted in the inset of Fig. 2. Here, a continuous increase of $-\rho_{\mathrm{d}} / \rho(H=0)$ between the transverse $\left(\Theta=90^{\circ}\right)$ and the longitudinal $\left(\Theta=0^{\circ}\right)$ orientation is observed. In a previous article we also reported on the temperature dependence of this reduced resistivity associated with DWs. ${ }^{7}$ For a $2 \mu \mathrm{m}$ wire in the longitudinal geometry $-\rho_{\mathrm{d}} / \rho(H=0)$ is about $1 \%$ at $1.5 \mathrm{~K}$ and disappears at $80 \mathrm{~K}$.

In order to correlate the stripe domain densities, which were measured at room temperature using a MFM with the observed low-temperature MR, the sample was warmed up to room temperature. At room temperature a known domain state was established by saturating the sample in transverse or longitudinal geometry and cooling in zero-applied field. After cooling, the resistivity and MR were unchanged. This is strong evidence that the domain configuration at $H=0$ is not altered by temperature.
In conclusion, using an epitaxial (110) Fe wire of $2 \mu \mathrm{m}$ linewidth, the DW density was varied continuously from 0.45 to $1.8 \mu \mathrm{m}$. In the presence of DWs a reduced resistivity was observed with a magnitude which depends on the DW density. This observation cannot be understood by means of a recently proposed mechanism in which spin-dependent electron scattering within a DW leads to a mixing of the minority- and majority-spin channels as in giant magnetoresistance. ${ }^{11}$ This mechanism would lead to an enhanced resistivity in the presence of DWs. More recently, weak localization has been proposed to explain such phenomena, ${ }^{12}$ but is not applicable in the observed temperature range at which electron-phonon scattering would destroy such coherence effects. It is possible that the interplay of orbital effects associated with spatially modulated internal magnetic fields in stripe and closure domains and in DWs with surface scattering may also be important. The internal magnetic-field direction within a DW, which is directed perpendicular to the surface of the wire, may lead to a reduced surface scattering of the carriers within the DW, and hence, to an enhanced conductivity in the presence of DWs. Nevertheless, at present the microscopic origin of this effect is unclear and further theoretical and experimental work is warranted.

This research was supported by DARPA-ONR Grant No. N00014-96-1-1207. The authors thank C. Noyan for Xray characterization and $\mathrm{M}$. Ofitserov for technical assistance. Microstructures were prepared at the CNF, Project No. 588-96.

${ }^{1}$ M. Viret, D. Vignoles, D. Cole, J. M. D. Coey, W. Allen, D. S. Daniel, and J. F. Gregg, Phys. Rev. B 53, 8464 (1996).

${ }^{2}$ J. F. Gregg, W. Allen, K. Ounadjela, M. Viret, M. Hehn, S. M. Thompson, and J. M. D. Coey, Phys. Rev. Lett. 77, 1580 (1996).

${ }^{3}$ K. Hong and N. Giordano, Phys. Rev. B 51, 9855 (1995).

${ }^{4}$ Y. Otani, K. Fukamichi, O. Kitakami, Y. Shimada, B. Pannetier, J. P. Nozieres, T. Matsuda, and A. Tonomura, Proceedings of the MRS Spring Meeting (San Francisco, 1997), Vol. 475, p. 215 (1997).

${ }^{5}$ T. R. Mc Guire and R. I. Potter, IEEE Trans. Magn. MAG-11, 1018 (1975).

${ }^{6}$ See, e.g., I. A. Campell and A. Fert, in Ferromagnetic Materials, edited by E. P. Wohlfarth (North-Holland, Amsterdam, 1982), Vol. 3.

${ }^{7}$ A. D. Kent, U. Ruediger, J. Yu, S. Zhang, P. M. Levy, and S. S. P. Parkin, IEEE Trans. Magn. 34, 900 (1998).

${ }^{8}$ U. Ruediger, J. Yu, S. Zhang, A. D. Kent, and S. S. P. Parkin, Phys. Rev. Lett. 80, 5639 (1998).

${ }^{9}$ B. M. Clemens, R. Osgood, A. P. Pyne, B. M. Lairson, S. Brennan, R. L. White, and W. D. Nix, J. Magn. Magn. Mater. 121, 37 (1993).

${ }^{10}$ C. Kittel, Phys. Rev. 70, 965 (1946).

${ }^{11}$ P. M. Levy and S. Zhang, Phys. Rev. Lett. 79, 5110 (1997).

${ }^{12}$ G. Tatara and H. Fukuyama, Phys. Rev. Lett. 78, 3773 (1997). 